\title{
Pseudolocality of Ricci Flow under Integral Bound of Curvature
}

\author{
Wang Yuanqi*
}

\begin{abstract}
We prove a pseudolocality type theorem for compact Ricci Flow under local integral bounds of curvature. The main tool is Local Ricci Flow introduced by Deane Yang in [4] and Pseudolocality Theorem of Perelman in [3]. We also study $L^{p}$ bounds for the derivatives of curvature and smooth extension of Local Ricci Flow.
\end{abstract}

\section{Introduction}

In this short paper we are interested in Pseudolocality phenomenon of compact Ricci Flow. In 1982, R.Hamilton introduced the Ricci flow in [1], where he deforms a Riemanniana metric in the direction of -2 times its Ricci curvature:

$$
\frac{\partial g_{i j}}{\partial t}=-2 R i c_{i j}
$$

Ricci flow gives a canonical way of deforming an arbitrary metric to a critical metric (Einstein metric in particular). This program of Ricci flow has been remarkably succesful in the recent years thanks to the seminal work of G. Perelman [3].

By the nature of this evolution equation, any change of initial metric initially and locally, will affect the evolution of metric afterwards globally. However, an elegant and powerful theorem by G. Perelman said that if the initial metric is closed to be Euclidean in some unit ball, then, no matter what happen far away, this almost Euclidean region will remains to be so for some fixed time. This gives us hope as how to control the metric based on local infomation even during a global flow. Following Perelman, we want to replace the almost Euclidean isoperimetric constant assumption in Perelman's original theorem by some integral bound of curvature.

Definition 1.1. $\digamma(n, p, K, \tau, \eta)$ assumption:

For all $n \geq 3, p>\frac{n}{2}, \eta, K$, there exists $\omega_{0}$ such that $\forall \tau \leq \omega_{0}$, We say a ball $[B(x, r), g]$ in a compact Riemannian Manifold satisfy $\digamma(n, p, K, \tau, \eta)$ assumption if :

1. $\operatorname{Vol} B(x, \tau r) \geq w(n)(\eta \tau r)^{n}$

\footnotetext{
*Department of Mathematics, UW-Madison. Email address: wang56@wisc.edu.
} 
2. $|R m|_{\frac{n}{2}[B(x, \tau r)]} \leq \varpi(n, \eta)$

3. $|\operatorname{Ric}|_{p[B(x, r)]} \leq K r^{\frac{n}{p}-2}, p>\frac{n}{2}$

In which

$$
\varpi(n, \eta)=\frac{7}{8} 2^{-1-\frac{2}{n}} \frac{(n-2)^{2}}{n^{2}(n-1)^{2}} \cdot \alpha^{2}(n-1) \cdot \alpha^{\frac{2}{n}-2}(n) \cdot(\eta)^{2 n+2}
$$

and $\alpha(n)\{\omega(n)\}$ is the volume of $n$-dim unit sphere $\{$ unit Euclidean ball $\}$.

The following is our main theorem:

Theorem 1.2. Pseudolocality of Compact Ricci Flow under Bounds on Curvature:

For all $n \geq 3, p>\frac{n}{2}, \eta, K, \tau$, there exists $\epsilon$ such that:

If $[g(t), M]$ is a compact solution to Ricci-Flow: $\partial_{t} g=-2$ Ric, , $0 \leq t \leq(\epsilon r)^{2}$ and when $t=0$ the ball $B_{0}\left(x_{0}, r\right)$ satisfies $\digamma(n, p, K, \tau, \eta)$ assumption and $R \geq-r^{2}$ in $B_{0}\left(x_{0}, r\right)$, then :

1. $|R m|(x) \leq \frac{1}{t}+(\epsilon r)^{-2}$

2. $\operatorname{VolB}\left(x, t^{\frac{1}{2}}\right) \geq C(n) t^{\frac{n}{2}}$

whenever $0<t \leq(\epsilon r)^{2}$ and $d_{t}\left(x, x_{0}\right) \leq \epsilon r$.

Remark 1.3. :

- This theorem is an $\epsilon$ - regularity type theorem for Ricci Flow. The $\epsilon$ - regularity theorem for Einstein 4-Manifold is well known, one could refer to Theorem 4.4 in [12] and Theorem 0.8 in [13] which are motivations of our work.

- The $\digamma(n, p, K, \tau, \eta)$ assumption ensure the assumption on isoperimetric constant in Perelman's Pseudolocality Theorem 6.1 to be valid. Assumption 1 and 2 in $\digamma(n, p, K, \tau, \eta)$ are definitely indispensable. The example in section 2 shows that assumption 2 and 3 in $\digamma(n, p, K, \tau, \eta)$ can not be simultaneously weaken.

According to Theorem 0.8 and section 11 in [13], one could expect to drop Assumption 1 in $\digamma$ if one works on the normalized Ricci Flow: $\partial_{t} g=-2 R i c+\frac{2 r}{n} g$. It is also interesting to investigate on whether Assumption 3 in $\digamma(n, p, K, \tau, \eta)$ could be dropped.

- Given a smooth compact Riemannian Manifold M, $\exists r_{0}$ sufficiently small such that the assumptions in $\digamma(n, p, K, \tau, \eta)$ are all valid in any ball of radius less that $r_{0}$.

- The reason why we need $n \geq 3$ is that we should use Moser Iteration to Theorem 6.3. The case $n=2$ should be similar with something modified, which we left to the interested reader to search on.

- The corresponding Finiteness of Diffeomorphism Types and Gromov-Hausdorff compactness of those manifolds satisfy the assumption of Theorem 1.2 are also valid. We will not state them here. 
The relationship between integral bounds on curvature and Isoperimetric constant has been studied for long. One could refer to the interesting works of [10, 8], 7], [4].

The invention of Local Ricci Flow really enable one to work on this more conveniently. In [4], using proper cutoff function $\chi$ on a Riemannian Manifold, Deane Yang considered the local deformation of the metric along Local Ricci Flow:

$$
\frac{\partial g}{\partial t}=-2 \chi^{2} R i c
$$

The short time existence of LRF is proved in [4], one could also refer to [5].

Under LRF, one could see some important informations on the local metric structure under local integral bounds of curvature. Most important of all, LRF is able to control the local integral norm of curvature $\int_{B(x . r)}|R m|^{\frac{n}{2}}$. Thus it's natural to use LRF to study the pseudolocality phenomenon of Global Ricci Flow.

In section 4 of this paper we study the smooth extension of the Local Ricci Flow. Namely, we provide the details of the $L^{p}$ bounds on derivative of curvature. It is unlikely to directly get bounds for $\int\left|\nabla^{m} R m\right|^{2}, m \geq 0$ as claimed in Theorem 9.2 in Page 93 of [4], the reason is illustrated in Theorem 4.1 and it's remark. Our method use induction which is based on $L^{p}$ estimate.

One might doubt whether LRF is necessary to prove Theorem 1.2 or not. We think the rescaling approach initiated in [11] might also work to get Proposition 3.13, which is crucial to Theorem 1.2. However, under the bound of $\mid$ Ric $\left.\right|_{p}, p>\frac{n}{2}$, the best possible regularity for the coordinates are $C^{1, \alpha}$ and $W^{3, p}$. Then one must argue the rescaled limit is flat using the assumption that $|R m|_{\frac{n}{2}}$ is sufficient before we take limit. This is challenging to me because the limit has little regularity. Anyway we think there might be an approach without LRF.

In a word, in this short paper we mainly prove Theorem 1.2 and study the smooth extension of Local Ricci Flow in section 5 to further explain Theorem 6.3 which is one of the main theorems in [4].

The arrangement of this paper is: In Section 2 we compute an example to get a feeling on this problem and see which assumptions are indispensable. In section 3 we prove Theorem 1.2. In Section 4 we do $L^{p}$ estimates and prove Theorem 4.1 on smooth extension of Local Ricci Flow.

Acknowlegdement: I would like to thank my advisor Chen Xiuxiong for introducing me to the interesting area of Pseudolocality of Ricci Flow and Local Ricci Flow. I would not be able to finish these without his useful discussions and encouragement. I am grateful to Wang Bing for reading this paper carefully and provide insightful comments. I also would like to thank Li Hao Zhao for reading my paper and Ye Li for reading section 4 carefully. 


\section{An Example}

I would like to point out that the conclusions of Theorem 1.2 will not hold if assumption 2 and 3 in $\digamma(n, p, K, \tau, \eta)$ are weakened. The counterexample comes from S. Angenent and D. Knopf [9].

More precisely, we have the following statement:

Claim 2.1. In dimension 4 , there is a family of Ricci-Flow $g_{G}(t), x_{G}$, constants $c, K$ independent of $G$ such that:

1. $|R m|_{2\left[B_{0}\left(x_{G}, c\right)\right]} \leq K$

2. $R \geq 0$ in $B_{0}\left(x_{G}, c\right)$

And $g_{G}(t)$ will become singular before time $G$.

Assume $g_{0}=d s^{2}+\psi^{2}(s) d S^{n}, s \in\left[-\frac{\pi}{2}, \frac{\pi}{2}\right]$ could be extended to be a smooth rotationally symmetric Riemannian metric on $S^{n+1}$. We define "necks" to be the local minimum of $\psi$, "bumps" to the local maximum of $\psi$. ("local" means the boundary points $-\frac{\pi}{2}$ and $\frac{\pi}{2}$ are not included). It is well know that:

Given a point $\mathrm{p}$, if $\left\{\frac{e_{1}}{\psi}, \ldots \frac{e_{n}}{\psi}\right\}$ is orthonomal at $\mathrm{p}$ and is tangent to the $S^{n}$ fibre, then:

$$
K_{N} \triangleq R\left(\partial s, \frac{e_{i}}{\psi}, \frac{e_{i}}{\psi}, \partial s\right)=-\frac{\ddot{\psi}}{\psi}, K_{T}=R\left(\frac{e_{j}}{\psi}, \frac{e_{i}}{\psi}, \frac{e_{i}}{\psi}, \frac{e_{j}}{\psi}\right)=\frac{1-(\dot{\psi})^{2}}{(\psi)^{2}}
$$

Definition 2.2. $a(s)=\psi^{2}\left(K_{N}-K_{T}\right), r_{\max } \triangleq|\psi(s)|$ where $\mathrm{s}$ is the smallest bump, and $r_{\text {min }} \triangleq|\psi(s)|$ where $\mathrm{s}$ is the smallest neck.

Then we could quote the theorem of S.Angenent and D.Knopf:

Theorem 2.3. Assume $g_{0}=d s^{2}+\psi^{2}(s) d S^{n}, s \in\left[-\frac{\pi}{2}, \frac{\pi}{2}\right]$ is a smooth rotationally symmetric Riemannian metric on $S^{n+1}$. Assume $g$ has one neck, at least one bump and satisfies:

1. $K_{T}>0$, or equivalently $|\dot{\psi}(s)|<1$.

2. $R>0$

3. $|a| \leq \mu$

4. $\frac{r_{\max }^{2}}{r_{\min }^{2}} \geq \frac{2 \mu+2 n}{n-1}$

Then $\exists \sigma_{1}(g), \sigma_{2}(g)$ such that if we evolve $g$ by Ricci-Flow:

$$
\partial_{t} g=-2 R i c
$$


before time $\frac{r_{\min (0)}^{2}}{n-1}$ we will have:

$$
r_{\min }(t) \rightarrow 0
$$

In particular, $K_{T}$ at the neck points ( the point might change along the flow) will tends to $+\infty$ before time $\frac{r_{\min (0)}^{2}}{n-1}$.

Furthermore, at the sigular time the volume of the whole manifold satisfy

$$
\sigma_{1}(g) \leq \operatorname{Vol}\left(g_{t}\right) \leq \sigma_{2}(g)
$$

Although it is trivial, I would like use the following things as an example of what happens:

Proposition 2.4. Theorem 1.2 will not hold if assumptions 2,3 in $\digamma(n, p, K, \tau, \eta)$ are simultaneously weaken.

Proof. Actually S.Angenent and D.Knopf constructed an example which satisfy the assumptions in Theorem 2.3. According to the examples, we could find a 4-dimensional example: $\exists c$ such that: $\psi$ is even function and when $s \leq c$ we have $g_{0}=d s^{2}+\left(G^{2}+s^{2}\right) d S^{3}$, for $\mathrm{G}$ sufficiently small. The global picture looks like a dumbell. We compute $\left|K_{N}\right|_{2},\left|K_{T}\right|_{2}$ to see:

Claim 2.5. when $G$ tend to 0

1. $\lim _{G \rightarrow 0}|R m|_{2[p|| s(p) \mid \leq c]}$ exists but is far bigger than the energy gap in assumption $\digamma(n, p, K, \tau, \eta)$.

2. $|\operatorname{Ric}|_{p[p|| s(p) \mid \leq c]}$ tends to $\infty, p>2$.

First: $K_{N}=-\frac{G^{2}}{\left(G^{2}+s^{2}\right)^{2}}$ and $K_{T}=\frac{G^{2}}{\left(G^{2}+s^{2}\right)^{2}}$ Then:

$$
\begin{aligned}
& \int_{(p|| s(p) \mid \leq c)}\left|K_{N}\right|^{2} d V o l \\
= & 2 \alpha(3) \int_{0}^{c}\left[\frac{G^{2}}{\left(G^{2}+s^{2}\right)^{2}}\right]^{2}\left(G^{2}+s^{2}\right)^{\frac{3}{2}} d s \\
\leq & 2^{\frac{7}{2}} \alpha(3) \int_{0}^{c} \frac{G^{4}}{(G+s)^{5}} \\
= & 2^{\frac{3}{2}} \alpha(3)\left[1-\frac{G^{4}}{(G+c)^{4}}\right]
\end{aligned}
$$

also we have:

$$
\int_{(p|| s(p) \mid \leq c)}\left|K_{N}\right|^{2} d V o l=\int_{(p|| s(p) \mid \leq c)}\left|K_{T}\right|^{2} d V o l
$$

Then:

$$
|R m|_{2}^{2} \leq 4 \cdot 6 \cdot 2^{\frac{3}{2}} \alpha(3)\left[1-\frac{G^{4}}{(G+c)^{4}}\right]
$$

Use $\alpha(3)=2 \pi^{2}$, we see:

$$
\lim _{G \rightarrow 0}|R m|_{2[p|| s(p) \mid \leq c]}=2^{\frac{11}{4}} \pi \sqrt{3}
$$


It means the Riemannian curvature has a concentration on the submanifold $(p \mid s(p)=0)$. Thus it's easy to see $|\operatorname{Ric}|_{p[p|| s(p) \mid \leq c]}$ tends to $\infty, p>2$.

One see that when $G \rightarrow 0$, the metric tensor goes to a cone pointwisely. Then the volume ratio around the center goes to $w(4)$.

Using $\alpha(3)=2 \pi^{2}, \alpha(4)=\frac{8 \pi^{2}}{3}$, in our case we see the energy gap $\varpi(4,1)$ in assumption $\digamma(n, p, K, \tau, \eta)$ is about $\frac{7}{8} \pi \cdot 3^{-\frac{1}{2}} 2^{-6}$. Thus $\lim _{G \rightarrow 0}|R m|_{2[p|| s(p) \mid \leq c]}>100 \varpi(4,1)$.

Thus we see the claim holds i.e: according to Theorem 2.3 of Angenent and Knopf, Ricci-Flow starting from $g_{0}=d s^{2}+\left(G+s^{2}\right) d S^{3}$ will develop singularity around the neck within time $G$. Since the metric is reflection symmetric, and the number of necks will not grow, we see that after the flow starts, the neck does not move, and the size of which will shrink to 0 . But c is fixed, thus when $\mathrm{G}$ is sufficiently small the solution does not satisfy the conclusion of Theorem 1.2,

Remark 2.6. Something about the Isoperimetric Constant in the above Example:

One see that when $G \rightarrow 0$, the metric goes to a cone in some sense. However, we can not say anything about the geometric convergence. I would like to point out that:

For those domain $\Omega_{N}$ which are bounded by noncontractible $S^{3}$ in $[p \mid s(p) \leq c]$, the quotients :

$$
\frac{\operatorname{Vol} l^{\frac{3}{4}}\left(\Omega_{N}\right)}{\operatorname{Vol}\left(\partial \Omega_{N}\right)}
$$

are uniformly bounded from above.

It's easy to see that it suffices to bound the above quotients for those domains whose boundary are level set of the variable $S$. We compute $\forall b$ :

$$
\begin{aligned}
& \operatorname{Vol}(p \mid 0 \leq s(p) \leq b) \\
\simeq & \int_{0}^{b}\left[G^{2}+s^{2}\right]^{\frac{3}{2}} d s \\
\simeq & {\left[(G+b)^{4}-G^{4}\right] }
\end{aligned}
$$

Thus consider the domain $\Omega_{b} \triangleq[p \mid s(p) \leq b]$, we have:

$$
\frac{\operatorname{Vol}^{\frac{3}{4}}(\Omega)}{\operatorname{Vol}(\partial \Omega)} \simeq \frac{\left[(G+b)^{4}-G^{4}\right]^{\frac{3}{4}}}{\left[G^{3}+b^{3}\right]}
$$

And $\frac{\left[(G+b)^{4}-G^{4}\right]^{\frac{3}{4}}}{\left[G^{3}+b^{3}\right]}$ is uniformly bounded from above independent of $G, b$. Then we see that for those domain $\Omega_{N}$ which are bounded by noncontractible $S^{3}$ in $[p \mid s(p) \leq c]$, the quotients :

$$
\frac{\operatorname{Vol} l^{\frac{3}{4}}\left(\Omega_{N}\right)}{\operatorname{Vol}\left(\partial \Omega_{N}\right)}
$$


are uniformly bounded from above. For those domain $\Omega_{C}$ which are bounded by contractible $S^{3}$ in $[p \mid s(p) \leq c]$, the quotients $\frac{\operatorname{Vol}^{\frac{3}{4}}\left(\Omega_{C}\right)}{\operatorname{Vol}\left(\partial \Omega_{C}\right)}$ are not known by me yet because there are very hyperbolic parts around the neck no matter how small $G$ is. However, I think it's also uniformly bounded independent of $\mathrm{G}$ and its own size and shape.

\section{Set up of Local Ricci flow and Proof of the Main Theorem}

Notation: $\Omega \Subset M$ means that $\Omega$ is a relatively compact domain of a manifold $M$ (M which might have boundary), $\partial \Omega$ is smooth and $\Omega \backslash \partial \Omega$ is open.

Definition 3.1. Suppose $[M, g]$ is a complete Riemannian Manifold, then the Local Ricci flow is the deformation of $\mathrm{g}$ in the direnction of $-2 \chi^{2}$ Ric, $\chi \in C_{0}^{\infty}(M)$ i.e Local Ricci Flow is the following Geometric PDE:

$$
\frac{\partial g}{\partial t}=-2 \chi^{2} \text { Ric, } \chi \in C_{0}^{\infty}(M)
$$

The short time existence of LRF is proved in [4]. One could also refer to [5] .

Definition 3.2. Tensor Lipshitz Distance:

Suppose $\left(M, g_{1}\right)$ and $\left(M, g_{2}\right)$ are two compact Riemannian Manifolds with boundary which are diffeomorphic to each other, denote $\dot{M}$ as the interior of $M$. Assume $g_{1}, g_{2}$ are nondegenerate on $\dot{M}$, i.e:

$$
\forall V_{p} \neq 0 \in T_{p} \dot{M}, p \in \dot{M} \text { we have } g_{i}\left(V_{p}, V_{p}\right)>0, i=1,2 .
$$

Then we define the Tensor Lipshitz Distance between $\left(M, g_{1}\right)$ and $\left(M, g_{2}\right)$ to be:

$$
d_{T L}\left[\left(M, g_{1}\right),\left(M, g_{2}\right)\right]=\sup _{p \in \dot{M}} \sup _{V_{p} \in T_{p} \dot{M}}\left|\log \frac{g_{1}\left(V_{p}, V_{p}\right)}{g_{2}\left(V_{p}, V_{p}\right)}\right|
$$

Remark 3.3. The notion of Tensor Lipshitz Distance is more flexible and local. $\left(M, g_{1}\right)$ and $\left(M, g_{2}\right)$ are not required to be geodesic convex. But if $\left(M, g_{1}\right)$ and $\left(M, g_{2}\right)$ are all geodesic convex in their complete manifolds, then Tensor Lipshitz Distance is equivalent to the ordinary Lipshitz Distance whose definition could be found in Green-Wu [6].

Lemma 3.4. For a linear space $R^{n}$, consider the Euclidean inner product $<,>$, and consider a nondegenerate symmetric matrix $g_{i j}$, then the following things are equavalent:

1. $g_{i j} v_{i} v_{j}$ is close to 1 for every $v$ s.t $|v|^{2}=1$ ( ||$^{2}$ is the Euclidean square norm).

2. The matrix $g_{i j}$ is close to the identity matrix $I$.

3. detg $_{i j}$ is close to 1 , and the determinant of $g$ on any codimension 1 linear subspace is close to 1 . 
Proof. $1 \longrightarrow 2$ :

Diagnalize $g$ using the orthogonal matrix h, namely:

$g=h \cdot \operatorname{Diag}\left(\lambda_{1}, \lambda_{2} \ldots \ldots \lambda_{n}\right) \cdot h^{t}$ in which $\operatorname{Diag}\left(\lambda_{1}, \lambda_{2} \ldots \ldots \lambda_{n}\right)$ is:

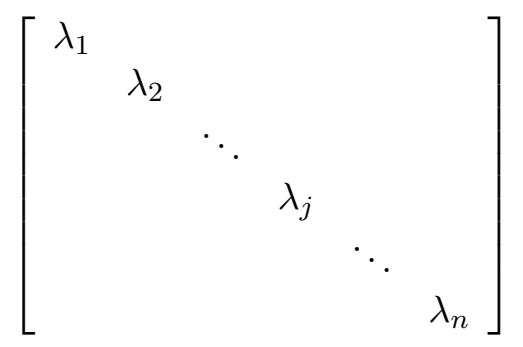

(1) says that $\lambda_{1}, \lambda_{2} \ldots \ldots, \lambda_{n}$ are all close to 1 , and because $\mathrm{h}$ is orthogonal then $|h|^{2}=t r h h^{t}=n$ and:

$$
|g-I|=\left|h(D-I) h^{t}\right| \leq n|D-I|
$$

then $\mathrm{g}$ is close to identity matrix.

$2 \longrightarrow 3$ :This is obvious.

$3 \longrightarrow 1$ : Denote $e_{j}$ is the eigenvector associated to $\lambda_{j}$, then we consider the hyperplane $\mathrm{H}$ perpendicular to $e_{j}$, then the determinant of $\mathrm{g}$ on $\mathrm{H}$ is:

$$
\lambda_{1}, \lambda_{2} \ldots \hat{\lambda j} \ldots \lambda_{n}
$$

in which $\hat{\lambda_{j}}$ means that the product is without $\lambda_{j}$. By the following:

$$
\lambda_{j}=\frac{\lambda_{1}, \lambda_{2} \ldots \lambda_{j} \ldots \lambda_{n}}{\lambda_{1}, \lambda_{2} \ldots \hat{\lambda}_{j} \ldots \lambda_{n}}
$$

Then the assumption that $\lambda_{1}, \lambda_{2} \ldots \hat{\lambda_{j} \ldots \lambda_{n}}$ and $\lambda_{1}, \lambda_{2} \ldots \lambda j \ldots \lambda_{n}$ are close to 1 tells us that $\lambda_{j}$ is close to $1 . \mathrm{j}$ is arbitrary, then we are done.

Lemma 3.5. If we have a sequence of positive definite matrix function $g_{(i j)}^{k}$ defined on $\dot{B}_{e}(R)$ which satisfy:

1. $g_{(i j)}^{k} \rightarrow g_{(i j)}^{\infty}$ uniformly on $\dot{B}_{e}(R), \forall i, j$

2. $\exists \lambda_{\min }>0$ such that $i n f_{k} i n f_{p \in \dot{B}_{e}(R)} g_{(i j)}^{k} \geq \lambda_{\min } I$

Then $g_{(i j)}^{k} \rightarrow g_{(i j)}^{\infty}$ w.r.t Tensor Lipshitz distance.

Proof. : For any vector field $v$ defined on $\dot{B}_{e}(R)$, denote $|v|^{2}$ to the Euclidean square norm. $\forall \epsilon$, in the coordinates we have:

$$
\left|g_{(m n)}^{\infty}-g_{(m n)}^{j}\right| \leq \epsilon
$$


Then:

$$
\begin{aligned}
& \frac{g^{\infty}(v, v)}{g^{j}(v, v)} \\
= & \frac{g_{(m n)}^{\infty} v^{m} v^{n}}{g_{j(m n)} v^{m} v^{n}} \\
\leq & \frac{g_{(m n)}^{j} v^{m} v^{n}+\epsilon|v|^{2}}{g_{(m n)}^{j} v^{m} v^{n}}, \quad|v|^{2}=\sum_{i=0}^{n} v_{i}^{2} \\
\leq & 1+\frac{\epsilon}{\lambda_{\min }}
\end{aligned}
$$

By the same reason we have:

$$
\frac{g^{\infty}(v, v)}{g^{j}(v, v)} \geq 1-\frac{\epsilon}{\lambda_{\min }}
$$

Proposition 3.6. $\forall \epsilon, \exists \delta$ If $\left(M, g_{1}\right),\left(M, g_{2}\right)$ satisfy

$$
d_{T L}\left\{\left(M, g_{1}\right),\left(M, g_{2}\right)\right\}<\delta
$$

Then the isoperimetric constant of $\left(M, g_{1}\right)$ and $\left(M, g_{2}\right)$ satisfy:

$$
\left|\log \left\{\frac{C_{s}\left(M, g_{1}\right)}{C_{s}\left(M, g_{2}\right)}\right\}\right|<\epsilon
$$

Proof. We first prove that: $\forall \epsilon, \exists \delta$ such that if $\left(M, g_{1}\right),\left(M, g_{2}\right)$ satisfy

$$
d_{T L}\left\{\left(M, g_{1}\right),\left(M, g_{2}\right)\right\}<\delta
$$

Then for any compact submanifold $S$ of M we have:

$$
\left|\log \left\{\frac{V \operatorname{lol}_{g_{1}} S}{\operatorname{Vol}_{g_{2}} S}\right\}\right|<\epsilon
$$

In particular, $\epsilon$ does not depend on the specific $S$ we choose. Next we start the proof. Given such a $S$, we could cut $S$ into $k$ pieces: $S=\bigcup_{i=1}^{k} S_{i}$ such that:

- $\operatorname{dim} S_{i} \cap S_{j} \leq \operatorname{dim} S-1$

- $\forall i, \exists r_{i}, x_{i}$ such that $B\left(x_{i}, r_{i}\right) \supset S_{i}$ and $r_{i}$ strictly smaller than:

$$
\min \left\{i n j_{\left.g_{1}\right|_{M}}\left(x_{i}\right), i n j_{\left.g_{2}\right|_{M}}\left(x_{i}\right), i n j_{\left.g_{1}\right|_{i}}\left(x_{i}\right), i n j_{g_{2} \mid S_{i}}\left(x_{i}\right), \operatorname{dist}_{g_{1}}\left(x_{i}, \partial M\right), \operatorname{dist}_{g_{2}}\left(x_{i}, \partial M\right)\right\}
$$

Then we have: 
Claim 3.7. $\forall$ i, if $d_{T L}\left(g_{1}, g_{2}\right)<\delta$, then:

$$
\left|\log \frac{\operatorname{Vol}_{g_{1}}\left(S_{i}\right)}{\operatorname{Vol}_{g_{2}}\left(S_{i}\right)}\right|<\frac{m \delta}{2}
$$

in which $m=\operatorname{dim}\left(S_{i}\right)$

Proof of the claim: In $B\left(x_{i}, r_{i}\right), \exists$ coordinates $\left(y_{1}, \ldots . y_{n}\right)$ such that:

$$
S_{i}=\left\{\left(y_{1}, \ldots . y_{n}\right) \mid y_{m+1}=. .=y_{n}=0\right\}
$$

Thus we use $\left(y_{1}, \ldots y_{m}\right)$ as coordinates for $S_{i}$.

$\forall p \in S_{i}$, choose $\left\{u_{1}, \ldots, u_{m}\right\}$ to be the normal coordinate of $\left\{g_{2}, S_{i}\right\}$ at $p$. Then $g_{2}\left(\frac{\partial}{\partial u_{i}}, \frac{\partial}{\partial u_{j}}\right)$ is the identity matrix at p. Thus by Lemma 3.4 and the assumption:

$$
\forall V_{p} \in T_{p} S_{i},\left|\log \frac{g_{1}\left(V_{p}, V_{p}\right)}{g_{2}\left(V_{p}, V_{p}\right)}\right| \leq \delta
$$

We know that:

$$
e^{-m \delta}<\operatorname{detg}_{1}\left(\frac{\partial}{\partial u_{i}}, \frac{\partial}{\partial u_{j}}\right)<e^{m \delta}
$$

Thus:

$$
e^{-\frac{m \delta}{2}}<\frac{\sqrt{\operatorname{detg}_{1}\left(\frac{\partial}{\partial u_{i}}, \frac{\partial}{\partial u_{j}}\right)}}{\sqrt{\operatorname{detg} g_{2}\left(\frac{\partial}{\partial u_{i}}, \frac{\partial}{\partial u_{j}}\right)}}<e^{\frac{m \delta}{2}}
$$

Then for any other coordinate system, in particular $\left(y_{1}, \ldots y_{m}\right)$, we have:

$$
e^{-\frac{m \delta}{2}}<\frac{\sqrt{\operatorname{det} g_{1}\left(\frac{\partial}{\partial y_{i}}, \frac{\partial}{\partial y_{j}}\right)}}{\sqrt{\operatorname{det} g_{2}\left(\frac{\partial}{\partial y_{i}}, \frac{\partial}{\partial y_{j}}\right)}}<e^{\frac{m \delta}{2}}
$$

then :

$$
e^{-\frac{m \delta}{2}}<\frac{\int_{D o m S_{i}} \sqrt{\operatorname{detg}_{1}\left(\frac{\partial}{\partial y_{i}}, \frac{\partial}{\partial y_{j}}\right)}}{\int_{D o m S_{i}} \sqrt{\operatorname{det} g_{2}\left(\frac{\partial}{\partial y_{i}}, \frac{\partial}{\partial y_{j}}\right)}}<e^{\frac{m \delta}{2}}
$$

which tells us

$$
e^{-\frac{m \delta}{2}}<\frac{\operatorname{Vol}_{g_{1}}\left(S_{i}\right)}{\operatorname{Vol}_{g_{2}}\left(S_{i}\right)}<e^{\frac{m \delta}{2}}
$$

So we've finished the proof of the claim.

Then we see:

$$
e^{-\frac{m \delta}{2}}<\frac{\sum_{i} \operatorname{Vol}_{g_{1}}\left(S_{i}\right)}{\sum_{i} \operatorname{Vol}_{g_{2}}\left(S_{i}\right)}<e^{\frac{m \delta}{2}}
$$

Which means:

$$
e^{-\frac{m \delta}{2}}<\frac{V o l_{g_{1}}(S)}{V o l_{g_{2}}(S)}<e^{\frac{m \delta}{2}}
$$


Then $\forall \Omega \Subset M$, using the above proof we see:

$$
e^{-(n-1) \delta}<\frac{\frac{V_{\operatorname{Vol}_{g_{1}}}(\partial \Omega)}{V o l_{g_{1}}(\Omega)^{\frac{n-1}{n}}}}{\frac{\operatorname{Vol}_{g_{2}}(\partial \Omega)}{V o l_{g_{2}}(\Omega)^{\frac{n-1}{n}}}}<e^{(n-1) \delta}
$$

Take $\delta=\frac{\epsilon}{n-1}$ which is independent of $\Omega$ then we are done.

Next we state two lemmas without proof.

Lemma 3.8. For all $n$ and $A, \exists c$ such that:

If $\left\{B_{p}(1), g\right\}$ is a n-dim Riemannian Unit Ball which satisfy: $A \cdot \operatorname{Vol} \Omega^{\frac{n-1}{n}} \leq \operatorname{Vol}(\partial \Omega), \forall \Omega \Subset$ $B(x, 1)$.

Then:

$$
\left\{\operatorname{VolB}{ }_{p}\left(\frac{3}{4}\right) \geq c\right\}
$$

Lemma 3.9. For all $n, A, \Lambda>0, \exists i_{0}$ such that: If $\left\{B_{p}(1), g\right\}$ is a n-dim Unit Ball in a complete Riemannian Manifold which satisfy:

1. $|R m| \leq \Lambda$

2. $A \cdot \operatorname{Vol}(\Omega)^{\frac{n-1}{n}} \leq \operatorname{Vol}(\partial \Omega), \forall \Omega \Subset B(x, 1)$

Then:

$$
\operatorname{inj}_{g}(p) \geq i_{0}
$$

Now we are ready to go on.

Proposition 3.10. For all $n, A$ and $\epsilon, \exists \omega, \delta$ such that:

If $\left\{B_{p}(1), g\right\}$ is a n-dim Unit Ball in a complete Riemannian Manifold which satisfy:

1. $|R m| \leq \delta$

2. $A \cdot \operatorname{Vol}(\Omega)^{\frac{n-1}{n}} \leq \operatorname{Vol}(\partial \Omega), \forall \Omega \Subset B(x, 1)$

Then $B_{p}(\omega)$ is within distance $\epsilon$ to a Euclidean Domain w.r.t Tensor Lipshitz Distance.

Proof. If not, by Lemma 3.9, $\exists \epsilon_{0}>0, \rho$ and a sequence of $\left\{B_{p_{j}}(1), g^{j}\right\}, \delta_{j} \rightarrow 0, \omega_{j} \rightarrow 0$ such that:

1. $|R m| \leq \delta_{j}$

2. $i n j_{g^{j}}\left(p_{j}\right) \geq \rho$

3. $B_{p_{j}}\left(\omega_{j}\right)$ stay further than $\epsilon_{0}$ to any Euclidean Domain w.r.t Tensor Lipshitz Distance.

Using harmonic coordinate theory in page 124 of [6], the main facts about harmonic balls in page 130 of [6], and the first inequality of the proof of the lemma in page 128 of [6], we have: $\exists$ constants $\sigma_{1}, \sigma_{2}, \sigma_{3}$ depending on $n, \rho$ such that: 
1. $\forall j, \exists \phi_{j}: B_{e}\left(\sigma_{1}\right) \rightarrow B_{p_{j}}(1)$ in which $\phi_{j}$ are harmonic coordinate system and $B_{e}\left(\sigma_{1}\right)$ is the Euclidean Ball with radius $\sigma_{1}$. Moreover:

$$
\left\|g_{k l}^{j}\right\|_{C^{1, \alpha}} \leq \operatorname{const}(\alpha, n, \rho)
$$

in which $g_{k l}^{j}=g^{j}\left(\frac{\partial}{\partial h_{k}^{j}}, \frac{\partial}{\partial h_{k}^{j}}\right)$ and $h_{1}^{j},,, h_{n}^{j}$ are coordinate functions of $\phi_{j}$. The $C^{1, \alpha}$ norm are also taken w.r.t the coordinate.

2. $B_{p_{j}}\left(\sigma_{3}\right) \subset \phi_{j}\left[B_{e}\left(\sigma_{1}\right)\right] \subset B_{p_{j}}\left(\sigma_{2}\right)$,

3. $\left|\phi_{j}^{*} g_{k l}^{j}-\delta_{k l}\right| \leq \frac{\operatorname{const}(n, \rho) \delta_{j}^{2}}{1-\operatorname{const}(n, \rho) \delta_{j}^{2}}$.

Thus $\phi_{j}^{*} g_{k l}^{j}$ tends to the Euclidean Metric on $B_{e}\left(\sigma_{1}\right)$ uniformly, then from lemma 3.5 we also know that: $\phi_{j}^{*} g_{k l}^{j}$ tends to the Euclidean Metric on $B_{e}\left(\sigma_{1}\right)$ w.r.t Tensor Lipshitz distance.

Thus it is easy to see that when $j$ is large enough $B_{p_{j}}\left(\sigma_{3}\right)$ will be within $\frac{\epsilon_{0}}{2}$ to a Euclidean Domain in $B_{e}\left(\sigma_{1}\right)$ w.r.t Tensor Lipshitz distance. Contradiction!.

Then we have:

Proposition 3.11. For all $n, A, \epsilon, \Lambda, \exists \hat{\omega}$ such that: If $\left\{B_{p}(1), g\right\}$ is a n-dim Unit Ball in a complete Riemannian Manifold which satisfy:

1. $|R m| \leq \Lambda$ in $B(x, 1)$.

2. $A \cdot \operatorname{Vol}(\Omega)^{\frac{n-1}{n}} \leq \operatorname{Vol}(\partial \Omega), \forall \Omega \Subset B(x, 1)$.

Then $B_{p}(\hat{\omega})$ is within distance $\epsilon$ to a Euclidean Domain w.r.t Tensor Lipshitz Distance.

Proof. This follows trivially from Proposition 3.10 and rescaling argument: If not, then $\exists \epsilon_{0}>$ 0 and $k \rightarrow \infty$ such that $B_{p}\left(\frac{1}{k}\right)$ stay further than $\epsilon_{0}$ to any Euclidean domain, then we rescale $\left[B_{p}\left(\frac{1}{k}\right), g\right]$ to be $\left[B_{p}(1), k^{\frac{1}{2}} g\right]$ which will satisfy the assumption of Proposition 3.10 if $k$ is large enough, which is a contradiction.

Proposition 3.12. For all $n \geq 3, p>\frac{n}{2}, A_{0}, K$ and $\epsilon$, there exists $v$ such that: Let $[g(t), B(x, 1)]$ be a n-dim complete Riemannian unit ball which satisfy :

1. $|f|_{\frac{2 n}{n-2}} \leq A_{0}|\nabla f|_{2}, \forall f \in C_{0}^{\infty}[B(x, 1)]$.

2. $|R m|_{\frac{n}{2}[B(x, 1)]} \leq \frac{\beta}{A_{0}^{2}}, \beta \leq \frac{1}{n^{2}}$

3. $|\operatorname{Ric}|_{p[B(x, 1)]} \leq K, p>\frac{n}{2}$

Then $B(x, v)$ is within distance $\epsilon$ to a Euclidean Domain w.r.t Tensor Lipshitz Distance. 
Proof. of Proposition 3.12, Denote $r(p)$ to be the distance function to x. Then choose:

$$
\hat{\chi}(p)=\left\{\begin{array}{cc}
1, & \text { if } 0 \leq r \leq \frac{1}{3} \\
2-3 r, & \text { if } \frac{1}{3} \leq r \leq \frac{2}{3} \\
0, & \text { if } \frac{2}{3} \leq r \leq 1
\end{array}\right.
$$

We see $\hat{\chi}$ is a lipshitz function with lipshitz constant $\operatorname{Lip}(\hat{\chi}) \leq 3$.

Then we could perturb $\hat{\chi}$ to be a function $\chi \in C_{0}^{\infty}[B(x, 1)]$ with $|\nabla \chi|_{\infty} \leq 4$ and:

$$
\chi \equiv 1 \text { in } B\left(x, \frac{1}{4}\right)
$$

Therefore we run the LRF:

$$
\frac{\partial g}{\partial t}=-2\left(\chi^{2} R i c\right)
$$

According to Theorem 6.3 we have:

$$
\left|\chi^{2} \operatorname{Ric}\right|_{\infty} \leq \bar{C}(n, p) A_{0}^{\frac{n}{p}} t^{-\frac{n}{2 p}}[t+1]^{\frac{n}{p}+\frac{2}{p}} K
$$

within a fixed time. Then $\exists t_{0}$ such that

$$
2 \int_{0}^{t_{0}}\left|\chi^{2} \operatorname{Ric}\right|_{\infty} \leq \frac{\epsilon}{2}
$$

Then $\forall v \in T \dot{B}(x, 1)$ we have:

$$
e^{-\frac{\epsilon}{2}} g(v, v) \leq g_{t_{0}}(v, v) \leq e^{\frac{\epsilon}{2}} g(v, v)
$$

Thus we have:

$$
d_{T L}\left\{\left[B(x, 1), g_{0}\right],\left[B(x, 1), g_{t_{0}}\right]\right\} \leq \frac{\epsilon}{2}
$$

(Here $B(x, 1)$ is the unit ball w.r.t the INITIAL METRIC).

By making $\epsilon$ small enough we could have :

$$
B\left(x, \frac{1}{16}\right) \subset B_{t_{0}}\left(x, \frac{1}{8}\right) \subset B\left(x, \frac{1}{4}\right)
$$

Therefore we have:

$$
\chi \equiv 1 \text { on } B_{t_{0}}\left(x, \frac{1}{8}\right)
$$

Thus from Theorem 6.3 we see:

- $|R m|_{\infty\left(B_{t_{0}}\left(x, \frac{1}{8}\right)\right)} \leq \operatorname{Const}(n) t_{0}^{-1}\left[t_{0}+1\right]^{\frac{4}{n}+2}$

- The isoperimetric constant in $\left[B_{t_{0}}\left(x, \frac{1}{8}\right), g_{t_{0}}\right]$ is less than $2 A_{0}$ if $\epsilon$ is small enough. 
Then according to Proposition $3.12, \exists \hat{v}$ such that : $B_{t_{0}}\left(x, \frac{\hat{v}}{8}\right)$ within distance $\frac{\epsilon}{2}$ to a Euclidean Domain w.r.t Tensor Lipshitz Distance. We denote the underlying Euclidean Domain to be $\Omega_{E}$.

Then we could see from $B\left(x, \frac{\hat{v}}{16}\right) \subseteq B_{t_{0}}\left(x, \frac{\hat{v}}{8}\right)$ and $d_{T L}\left\{\left[B(x, 1), g_{0}\right],\left[B(x, 1), g_{t_{0}}\right]\right\}<\frac{\epsilon}{2}$ (Here $B(x, 1)$ is the unit ball w.r.t the INITIAL METRIC) that $\exists$ a Euclidean domain $\hat{\Omega}_{E} \subset \Omega_{E}$ s.t:

$$
d_{T L}\left\{B\left(x, \frac{\hat{v}}{16}\right), \hat{\Omega}_{E}\right\}<\frac{\epsilon}{2}+\frac{\epsilon}{2}=\epsilon
$$

Then we are ready to prove the following:

Proposition 3.13. Strong Noncollapsed:

For all $n \geq 3, p>\frac{n}{2}, \eta, K, \tau, \epsilon$, there exists $\sigma$ such that:

If $[g, B(x, 1)]$ is a unit Ball in an complete $n$-dim smooth Riemannian manifold and satisfies $\digamma(n, p, K, \tau, \eta)$ assumption, then :

$$
(1-\epsilon) C_{E} \cdot \operatorname{Vol}(\Omega)^{\frac{n-1}{n}} \leq \operatorname{Vol}(\partial \Omega), \forall \Omega \Subset B(x, \sigma)
$$

In which $C_{E}$ is the isoperimetric constant of the Euclidean space.

Proof. This is directly implied by Theorem 6.2, Proposition 3.12 and 3.6. What is interesting is the computation of the constant $\varpi(n, \eta)$. Notice that $L^{2}$ Sobolev constant in Theorem 6.3 is $A_{0}=\frac{2 n-2}{n-2} C_{s}^{-1}, C_{s}$ is the underlying isoperimetric constant which can be found in Theorem 6.2. Furthermore the $C_{s}$ in Theorem 6.2 is close to the isoperimetric constant of the upper hemisphere.

Now we are ready to prove Theorem 1.2 .

Proof. of Theorem 1.2

It suffices to prove the case: $r=1$. By proposition 3.13 we see that $\exists \delta$ such that the assumption of almost Euclidean isoperimetrc constant in Perelman's theorem holds in a smaller ball $B\left(x_{0}, \delta\right)$. Then using Perelman's Theorem 6.1, we see that the conclusion of Theorem 6.1 holds in a even smaller ball: $B_{t}\left(x_{0}, \hat{\epsilon}\right), \hat{\epsilon}=\epsilon \delta$ in which $\epsilon$ is exact the one in Theorem 6.1.

\section{Extention of Local Ricci Flow under Pointwise Bound of Curvature}

This section is devoted to the proof of Theorem 4.1. We just provide the details of the smooth extension part of Theorem 6.3 which is one of the main theorems in [4].

Theorem 4.1. Long Time Existence of LRF:

Suppose $\left\{g(t), M, \chi \in C_{0}^{\infty}(M), t \in[0, T)\right\}$ is a n-dim solution to Local Ricci Flow and $T<\infty$. If $\left|\chi^{2} R m\right|_{\infty} \leq C, C$ is independent of $t$, then the solution could be extended smoothly beyond $T$. 
Remark 4.2. The reason why Theorem 4.1 holds is:

Fix $m, p, \exists \dot{p}, \ddot{p}, \breve{p}$ such that:

1. $\left|\nabla^{m}\left(\chi^{2} R m\right)\right|_{p}$ depend on $\left|\nabla^{m-1}(R m)\right|_{\dot{p}}$ as highest order term.

2. $\left|\nabla^{m-1}(R m)\right|_{\dot{p}}$ depend on $\left|\nabla^{m} \chi\right|_{\ddot{p}}$ as highest order term.

3. $\left|\nabla^{m} \chi\right|_{\ddot{p}}$ depend on $\left|\nabla^{m-1}\left(\chi^{2} R m\right)\right|_{\breve{p}}$ as highest order term.

Then we could use induction to control $\left|\nabla^{m}\left(\chi^{2} R m\right)\right|_{p}, \forall p$.

We first notice the following extension proposition.

Proposition 4.3. If $g(t), t \in[0, T)$ is a solution to Local Ricci Flow on $\Omega$ :

$$
\frac{\partial g}{\partial t}=-2\left(\chi^{2} R i c\right), \chi \in C_{0}^{\infty}(\Omega)
$$

Then if $\forall m \geq 0,\left|\nabla^{m}\left(\chi^{2} R m\right)\right|_{\infty}$ is uniformly bounded on $[0, T)$, then the Local Ricci Flow could be extended smoothly through $T$.

Proof. We use the argument of Hamilton in [2]. What is different is that here $\nabla^{m}\left(\chi^{2} R m\right)$ plays the crucial role.

Next we compute the evolution for $R m$ :

$$
\begin{aligned}
& \frac{\partial R_{i j k l}}{\partial t} \\
= & \chi^{2}\left[\Delta R_{i j k l}\right. \\
+ & \left(R_{i j p q} R_{p q k l}+2 R_{i p k q} R_{j p l q}-2 R_{i p l q} R_{j p k q}\right) \\
- & \left.\left(R_{i p} R_{p j k l}+R_{j p} R_{i p k l}+R_{k p} R_{i j p l}+R_{l p} R_{i j k p}\right)\right] \\
- & {\left[\left(\nabla_{i} \nabla_{k} \chi^{2}\right) R_{j l}+\left(\nabla_{j} \nabla_{l} \chi^{2}\right) R_{i k}-\left(\nabla_{i} \nabla_{l} \chi^{2}\right) R_{j k}-\left(\nabla_{j} \nabla_{k} \chi^{2}\right) R_{i l}\right] } \\
- & {\left[\left(\nabla_{i} \chi^{2}\right) \nabla_{k} R_{j l}+\left(\nabla_{k} \chi^{2}\right) \nabla_{i} R_{j l}+\left(\nabla_{j} \chi^{2}\right) \nabla_{l} R_{i k}+\left(\nabla_{l} \chi^{2}\right) \nabla_{j} R_{i k}\right.} \\
- & \left.\left(\nabla_{j} \chi^{2}\right) \nabla_{k} R_{i l}-\left(\nabla_{k} \chi^{2}\right) \nabla_{j} R_{i l}-\left(\nabla_{l} \chi^{2}\right) \nabla_{i} R_{j k}-\left(\nabla_{i} \chi^{2}\right) \nabla_{l} R_{j k}\right]
\end{aligned}
$$

First, let $T$ and $S$ be two tensors, I would like to use the " $T * S$ " to denote all possible algebraic tensor product between them, just for convenience. And here "*" is not necessarily unimodular, i.e $|T * S| \leq|T| \cdot|S|$ does not necessarily holds. Second, please notice that unless otherwise stated, everything depends on time.

\section{Lemma 4.4.}

$$
\begin{aligned}
& \frac{\partial \nabla^{m} R m}{\partial t} \\
= & \chi^{2} \Delta \nabla^{m} R m+\sum_{i+j+k=m+2, k \leq m+1}^{m} \nabla^{i} \chi * \nabla^{j} \chi * \nabla^{k} R m \\
+ & \sum_{i+j+k=m}^{m} \nabla^{i}\left(\chi^{2}\right) * \nabla^{j} R m * \nabla^{k} R m
\end{aligned}
$$


Proof. We use induction argument here. Suppose for $m-1$ the conclusion holds, then we have:

$$
\begin{aligned}
& \frac{\partial \nabla \nabla^{m-1} R m}{\partial t} \\
= & \nabla^{m-1} R m * \nabla\left(\chi^{2} R i c\right)+\nabla\left[\chi^{2} \Delta \nabla^{m-1} R m\right] \\
+ & \nabla\left[\sum_{i+j+k=m+1, k \leq m} \nabla^{i} \chi * \nabla^{j} \chi * \nabla^{k} R m\right] \\
+ & \nabla\left[\sum_{i+j+k=m-1} \nabla^{i}\left(\chi^{2}\right) * \nabla^{j} R m * \nabla^{k} R m\right]
\end{aligned}
$$

Notice that

$$
\begin{aligned}
& \nabla\left[\chi^{2} \Delta \nabla^{m-1} R m\right] \\
= & \chi^{2} \Delta \nabla^{m} R m+\chi^{2} \nabla^{m-1} R m * \nabla R m \\
+ & \chi^{2} \nabla^{m} R m * R m+\left(\nabla \chi^{2}\right) * \Delta \nabla^{m-1} R m
\end{aligned}
$$

If we consider

$$
\nabla^{m-1} R m * \nabla\left(\chi^{2} R i c\right), \chi^{2} \nabla^{m-1} R m * \nabla R m, \chi^{2} \nabla^{m} R m * R m
$$

as the terms in $\nabla\left[\sum_{i+j+k=m-1} \nabla^{i}\left(\chi^{2}\right) * \nabla^{j} R m * \nabla^{k} R m\right]$ and

$$
\left(\nabla \chi^{2}\right) * \Delta \nabla^{m-1}(R m)
$$

as one of the terms in

$$
\nabla\left[\sum_{i+j+k=m+1, k \leq m} \nabla^{i} \chi * \nabla^{j} \chi * \nabla^{k} R m\right]
$$

then we are done.

In the same way we could see:

\section{Lemma 4.5.}

$$
\begin{aligned}
& \frac{\partial \nabla^{m}\left(\chi^{2} R m\right)}{\partial t} \\
= & \chi^{2} \Delta \nabla^{m}\left(\chi^{2} R m\right)+\sum_{i+j+k=m+2, k \leq m+1}^{m} \nabla^{i} \chi * \nabla^{j} \chi * \nabla^{k}\left(\chi^{2} R m\right) \\
+ & \sum_{\substack{i+j+k=m, k \leq m-1 \\
+}}^{m} \nabla^{i}\left(\chi^{2}\right) * \nabla^{j}\left(\chi^{2} R m\right) * \nabla^{k} R m \\
& \chi^{2} R\left(\chi^{2} R m\right)
\end{aligned}
$$




\section{Lemma 4.6.}

$$
\frac{\partial}{\partial t}\left|\nabla^{m} \chi\right| \leq C(n, k) \sum_{k \leq m-1}\left|\nabla^{k}\left(\chi^{2} R i c\right)\right| \cdot\left|\nabla^{m-k} \chi\right|
$$

And we should state the following lemmas:

Lemma 4.7. If $f$ and $g$ are nonnegative functions of $t, t \in[0, T), b$ is a nonnegative constant. Assume $f$ satisfy:

$$
\frac{d f}{d t} \leq g+b f
$$

Then on $[0, T)$ we have:

$$
f \leq\left[f(0)+\int_{0}^{t} e^{-b s} g d s\right] e^{b t}
$$

Lemma 4.8. If $y(t)$ is nonnegative functions of $t, t \in[0, T)$. $a, b$ are nonnegative constants. Assume y satisfy:

$$
\frac{d y}{d t} \leq a \int_{0}^{t} y d s+b y+1
$$

Then $\exists w, k$ which depend on $a, b$ and $y(0)$ such that:

$$
y \leq w e^{k t}, \forall t \in[0, T) .
$$

Proof. It suffices to choose $w, k$ large enough to ensure:

$$
\left(w e^{k t}\right)^{\prime}>a \int_{0}^{t}\left(w e^{k t}\right) d s+b\left(w e^{k t}\right)+1 \text { and } w>y(0)
$$

to get the comparison function.

Proposition 4.9. If $\left|\chi^{2} R m\right|_{\infty}$ is uniformly bounded along a given solution of LRF $g(t), t \in$ $[0, T)$, then $|\nabla \chi|_{\infty}$ and the Sobolev constant $A$ are uniformly bounded on $[0, T)$.

Proof. One only have to notice that the metrics $g(t), t \in[0, T)$ are uniformly equivalent to each other.

From now we will do curvature estimate. I will denote all the quantities which are uniformly bounded independent of time on $[0, T)$ by $C$. The $C$ in different places may have different meaning, but it should be clear from the context that what does the $C$ represent. If some quantity is assumed to be uniformly bounded on $[0, T)$ in a specific lemma, I would also denote it by $C$.

Lemma 4.10. $\forall p>100$. If $g(t), t \in[0, T)$ is a smooth solution to $L R F$ on $M$ and satisfy: $\left|\chi^{2} R m\right|_{\infty}$ and $|\nabla \chi|_{\infty}$ are uniformly bounded on $[0, T)$, then $|R m|_{p}$ is uniformly bounded on $[0, T)$. 
Proof. Recall that

$$
\begin{aligned}
& \frac{\partial}{\partial t} \int|R m|^{p} \\
= & p\left\{-\int \chi^{2}|\nabla R m|^{2}|R m|^{p-2}+\int|R m|^{p-2}<\chi \nabla \chi * \nabla R m, R m>\right. \\
+ & \int|R m|^{p-2}<\chi^{2} R m * R m, R m> \\
+ & \left.\int|R m|^{p-2}<\nabla \chi * \nabla \chi * R m, R m>+\int|R m|^{p-2}<\chi \nabla^{2} \chi * R m, R m>\right\}
\end{aligned}
$$

Apply integration by parts to last term yields:

$$
\begin{aligned}
& \int|R m|^{p-2}<\chi \nabla^{2} \chi * R m, R m> \\
= & -(p-2) \int|R m|^{p-4}<\nabla R m, R m><\chi \nabla \chi * R m, R m> \\
+ & \int|R m|^{p-2}<\chi \nabla \chi * \nabla R m, R m> \\
+ & \int|R m|^{p-2}<\nabla \chi * \nabla \chi * R m, R m> \\
+ & \int|R m|^{p-2}<\chi \nabla \chi * R m, \nabla R m> \\
\leq & C \int|R m|^{p}+\frac{1}{16} \int \chi^{2}|\nabla R m|^{2}|R m|^{p-2}
\end{aligned}
$$

In which we used:

$$
\begin{aligned}
& \int|R m|^{p-2}<\chi \nabla \chi * \nabla R m, R m> \\
\leq & C \int|R m|^{p}+\frac{1}{16} \int \chi^{2}|\nabla R m|^{2}|R m|^{p-2}
\end{aligned}
$$

Then:

$$
\frac{\partial}{\partial t} \int|R m|^{p} \leq C \int|R m|^{p}
$$

Thus $|R m|_{p}$ is uniformly bounded on $[0, T)$.

Then we are ready to bound the integral of the first derivative of curvature:

Lemma 4.11. $\forall p>100$. If $g(t), t \in[0, T)$ is a smooth solution to LRF on $M$ assume further on $[0, T)$ :

- $|R m|_{q}$ is uniformly bounded, $\forall q>100$. 
- $\left|\chi^{2} R m\right|_{\infty}$ and $|\nabla \chi|_{\infty}$ are uniformly bounded.

Then we have: $\left|\nabla\left(\chi^{2} R m\right)\right|_{p}$ and $\left|\nabla^{2} \chi\right|_{p}$ are uniformly bounded on $[0, T)$.

Remark 4.12. The reason why we should do this lower order estimate first is $\left|\nabla^{2} \chi\right|_{q}$ and $\left|\nabla\left(\chi^{2} R m\right)\right|_{q}$ are interplaying along the flow, and both of them are crucial for us to go on with induction!

Proof. First from lemma 4.7 we have:

$$
\left|\nabla^{2} \chi\right|_{p} \leq C\left[\left|\nabla^{2} \chi\right|_{p}(0)+\int_{0}^{t}\left|\nabla\left(\chi^{2} R i c\right)\right|_{p} d s\right]
$$

So we could see:

$$
\frac{\partial \int\left|\nabla\left(\chi^{2} R m\right)\right|^{p}}{\partial t} \leq p \sum_{i=0}^{9} I_{i} .
$$

- $I_{0}=-\int \chi^{2}\left|\nabla^{2}\left(\chi^{2} R m\right)\right|^{2}\left|\nabla\left(\chi^{2} R m\right)\right|^{p-2}$

- $I_{1}=\int\left|\nabla\left(\chi^{2} R m\right)\right|^{p-2}<\nabla\left(\chi^{2} R m\right), \chi * \nabla^{3} \chi *\left(\chi^{2} R m\right)>$

- $I_{2}=\int\left|\nabla\left(\chi^{2} R m\right)\right|^{p-2}<\nabla\left(\chi^{2} R m\right), \chi * \nabla^{2} \chi * \nabla\left(\chi^{2} R m\right)>$

- $I_{3}=\int\left|\nabla\left(\chi^{2} R m\right)\right|^{p-2}<\nabla\left(\chi^{2} R m\right), \nabla \chi * \nabla \chi * \nabla\left(\chi^{2} R m\right)>$

- $I_{4}=\int\left|\nabla\left(\chi^{2} R m\right)\right|^{p-2}<\nabla\left(\chi^{2} R m\right), \nabla \chi * \nabla^{2} \chi *\left(\chi^{2} R m\right)>$

- $I_{5}=\int\left|\nabla\left(\chi^{2} R m\right)\right|^{p-2}<\nabla\left(\chi^{2} R m\right), \chi \nabla \chi * \chi^{2} R m * R m>$

- $I_{6}=\int\left|\nabla\left(\chi^{2} R m\right)\right|^{p-2}<\nabla\left(\chi^{2} R m\right), \chi^{2} R m * \nabla\left(\chi^{2} R m\right)>$

- $I_{7}=\int\left|\nabla\left(\chi^{2} R m\right)\right|^{p-2}<\nabla\left(\chi^{2} R m\right), \chi \nabla \chi * \nabla^{2}\left(\chi^{2} R m\right)>$

It's trivial to see that:

$$
\begin{gathered}
I_{5} \leq C\left|\nabla\left(\chi^{2} R m\right)\right|_{p}^{p-1} \\
I_{3}, I_{6} \leq C\left|\nabla\left(\chi^{2} R m\right)\right|_{p}^{p} \\
I_{4} \leq C\left|\nabla\left(\chi^{2} R m\right)\right|_{p}^{p-1}\left|\nabla^{2} \chi\right|_{p}
\end{gathered}
$$

The biggest trouble come from $I_{1}, I_{2}$ because they involve second and third derivatives of $\chi$. First we estimate $I_{2}$ :

$$
\begin{array}{ll}
I_{2} & =-\int\left|\nabla\left(\chi^{2} R m\right)\right|^{p-2}<\nabla^{2}\left(\chi^{2} R m\right), \chi \nabla \chi * \nabla\left(\chi^{2} \text { Ric }\right)> \\
- & \int\left|\nabla\left(\chi^{2} R m\right)\right|^{p-2}<\nabla\left(\chi^{2} R m\right), \chi \nabla \chi * \nabla^{2}\left(\chi^{2} R i c\right)> \\
- & \int\left|\nabla\left(\chi^{2} R m\right)\right|^{p-2}<\nabla\left(\chi^{2} R m\right), \nabla \chi * \nabla \chi * \nabla\left(\chi^{2} R i c\right)> \\
-(p-2) & \int\left|\nabla\left(\chi^{2} R m\right)\right|^{p-4}<\nabla^{2}\left(\chi^{2} R m\right), \nabla\left(\chi^{2} R m\right)><\nabla\left(\chi^{2} R m\right), \chi \nabla \chi * \nabla\left(\chi^{2} R i c\right)>
\end{array}
$$


Then we estimate:

$$
\begin{array}{r}
\left.\left|-\int\right| \nabla\left(\chi^{2} R m\right)\right|^{p-2}<\nabla^{2}\left(\chi^{2} R m\right), \chi \nabla \chi * \nabla\left(\chi^{2} R i c\right)>\mid \\
\leq \frac{1}{16} \int \chi^{2}\left|\nabla^{2}\left(\chi^{2} R m\right)\right|^{2}\left|\nabla\left(\chi^{2} R m\right)\right|^{p-2}+C \int\left|\nabla\left(\chi^{2} R m\right)\right|^{p}
\end{array}
$$

Estimate the other terms in the same way we have:

$$
I_{2} \leq \frac{1}{8} \int \chi^{2}\left|\nabla^{2}\left(\chi^{2} R m\right)\right|^{2}\left|\nabla\left(\chi^{2} R m\right)\right|^{p-2}+C \int\left|\nabla\left(\chi^{2} R m\right)\right|^{p}
$$

Then we estimate $I_{1}$ :

$$
\begin{aligned}
I_{1}= & -\int\left|\nabla\left(\chi^{2} R m\right)\right|^{p-2}<\nabla^{2}\left(\chi^{2} R m\right), \chi \nabla^{2} \chi *\left(\chi^{2} R i c\right)> \\
-(p-2) & \int\left|\nabla\left(\chi^{2} R m\right)\right|^{p-4}<\nabla^{2}\left(\chi^{2} R m\right), \nabla\left(\chi^{2} R m\right)><\nabla\left(\chi^{2} R m\right), \chi \nabla^{2} \chi *\left(\chi^{2} R i c\right)> \\
- & \int\left|\nabla\left(\chi^{2} R m\right)\right|^{p-2}<\nabla\left(\chi^{2} R m\right), \nabla \chi * \nabla^{2} \chi *\left(\chi^{2} R i c\right)> \\
- & \int\left|\nabla\left(\chi^{2} R m\right)\right|^{p-2}<\nabla\left(\chi^{2} R m\right), \chi \nabla^{2} \chi * \nabla\left(\chi^{2} R i c\right)>
\end{aligned}
$$

Notice that:

$$
\begin{aligned}
& \left.\left|-\int\right| \nabla\left(\chi^{2} R m\right)\right|^{p-2}<\nabla^{2}\left(\chi^{2} R m\right), \chi \nabla^{2} \chi *\left(\chi^{2} R i c\right)>\mid \\
\leq & \frac{1}{16} \int \chi^{2}\left|\nabla^{2}\left(\chi^{2} R m\right)\right|^{2}\left|\nabla\left(\chi^{2} R m\right)\right|^{p-2}+C \int\left|\nabla^{2} \chi\right|^{2}\left|\left(\chi^{2} R i c\right)\right|^{2}\left|\nabla\left(\chi^{2} R m\right)\right|^{p-2}
\end{aligned}
$$

and also

$$
\begin{aligned}
& (p-2) \int\left|\nabla\left(\chi^{2} R m\right)\right|^{p-4}<\nabla^{2}\left(\chi^{2} R m\right), \nabla\left(\chi^{2} R m\right)><\nabla\left(\chi^{2} R m\right), \chi \nabla^{2} \chi *\left(\chi^{2} R i c\right)> \\
\leq & \frac{1}{16} \int \chi^{2}\left|\nabla^{2}\left(\chi^{2} R m\right)\right|^{2}\left|\nabla\left(\chi^{2} R m\right)\right|^{p-2}+C\left|\nabla^{2} \chi\right|_{p}^{2}\left|\nabla\left(\chi^{2} R m\right)\right|_{p}^{p-2}
\end{aligned}
$$

The other two terms in $I_{1}$ are the same as those in $I_{2}, I_{4}$. Thus we get:

$$
\begin{aligned}
& I_{1} \\
\leq & \frac{1}{16} \int \chi^{2}\left|\nabla^{2}\left(\chi^{2} R m\right)\right|^{2}\left|\nabla\left(\chi^{2} R m\right)\right|^{p-2} \\
+ & C\left|\nabla^{2} \chi\right|_{p}^{2}\left|\nabla\left(\chi^{2} R m\right)\right|_{p}^{p-2} \\
+ & C\left|\nabla^{2} \chi\right|_{p}\left|\nabla\left(\chi^{2} R m\right)\right|_{p}^{p-1}+C\left|\nabla\left(\chi^{2} R m\right)\right|_{p}^{p}
\end{aligned}
$$


The estimate of $I_{7}$ is the same as the technipue of one of the terms in $I_{2}$. Then the conclusion is:

$$
\begin{aligned}
& \frac{\partial \int\left|\nabla\left(\chi^{2} R m\right)\right|^{p}}{\partial t} \\
= & \left\{C\left|\nabla\left(\chi^{2} R m\right)\right|_{p}^{p-2}\left[\left|\nabla^{2} \chi\right|_{p}(0)+\int_{0}^{t}\left|\nabla\left(\chi^{2} R i c\right)\right|_{p} d s\right]^{2}\right. \\
+ & C\left|\nabla\left(\chi^{2} R m\right)\right|_{p}^{p-1}\left[\left|\nabla^{2} \chi\right|_{p}(0)+\int_{0}^{t}\left|\nabla\left(\chi^{2} R i c\right)\right|_{p} d s\right] \\
+ & C\left|\nabla\left(\chi^{2} R m\right)\right|_{p}^{p-1}+C \int\left|\nabla\left(\chi^{2} R m\right)\right|^{p}
\end{aligned}
$$

Then we use Schwarz inequality and Holder Inequality to see:

$$
\frac{d}{\partial t}\left|\nabla\left(\chi^{2} R m\right)\right|_{p}^{2} \leq C \int_{0}^{t}\left|\nabla\left(\chi^{2} R m\right)\right|_{p}^{2} d s+C\left|\nabla\left(\chi^{2} R m\right)\right|_{p}^{2}+1
$$

Then from lemma 4.8 we see that $\left|\nabla\left(\chi^{2} R m\right)\right|_{p}^{2}$ is uniformly bounded on $[0, T)$, thus $\left|\nabla^{2} \chi\right|_{p}$ is also uniformly bounded on $[0, T)$.

Proposition 4.13. If $g(t), t \in[0, T)$ is a smooth solution to LRF on M. Assume on $[0, T)$ we have:

1. $\forall 0 \leq i \leq m+1,\left|\nabla^{i} \chi\right|_{q}$ are uniformly bounded, $\forall q>100$

2. $\forall q>100,|R m|_{q}$ is uniformly bounded.

3. $\left|\chi^{2} R m\right|_{\infty}$ and $|\nabla \chi|_{\infty}$ are uniformly bounded.

Then $\forall p>100,\left|\nabla^{m} R m\right|_{p}$ is uniformly bounded on $[0, T)$.

Proof. We argue by induction. When $m=0$ the conclusion is right. For all $m \geq 1$, assume that $\forall 0 \leq k \leq m-1, p>100$ :

$$
\int\left|\nabla^{k} R m\right|^{p}
$$

is uniformly bounded. Then we compute:

$$
\begin{aligned}
& \frac{\partial \int\left|\nabla^{m} R m\right|^{p}}{\partial t} \\
\leq & C\left\{\sum_{i+j+k=m+2, i, j, k \leq m+1} \int\left|\nabla^{i} \chi\right|\left|\nabla^{j} \chi \| \nabla^{k}(R m)\right|\left|\nabla^{m} R m\right|^{p-1}\right. \\
+ & \sum_{i+j+k=m} \int\left|\nabla^{i}\left(\chi^{2}\right)\right|\left|\nabla^{j} R m\right|\left|\nabla^{k}(R m)\right|\left|\nabla^{m} R m\right|^{p-1} \\
+ & \left.\int\left|\nabla^{m} R m\right|^{p-2}<\nabla^{m} R m, \chi \nabla^{m+2} \chi * R m>\right\} \\
- & p \int \chi^{2}\left|\nabla^{m+1} R m\right|^{2}\left|\nabla^{m} R m\right|^{p-2}
\end{aligned}
$$


Apply integration by parts to the term $\int\left|\nabla^{m} R m\right|^{p-2}<\nabla^{m} R m, \chi \nabla^{m+2} \chi * R m>$ and use the same technique as we did in Lemma 4.11 (integration by parts, Schwarz Inequality and holder inequality) as well as the induction hypothesis to see:

$$
\begin{aligned}
& \frac{\partial \int\left|\nabla^{m} R m\right|^{p}}{\partial t}+\frac{p}{2} \int \chi^{2}\left|\nabla^{m+1} R m\right|^{2}\left|\nabla^{m} R m\right|^{p-2} \\
\leq & C \int\left|\nabla^{m} R m\right|^{p}+C\left|\nabla^{m} R m\right|_{p}^{p-1}+C
\end{aligned}
$$

then $\int\left|\nabla^{m} R m\right|^{p}$ is uniformly bounded on $[0, T)$.

Proposition 4.14. . If $g(t), t \in[0, T)$ is a smooth solution to LRF on $M$ and assume on $[0, T)$ the following hold:

- $\forall q>100,|R m|_{q},\left|\nabla^{2} \chi\right|_{q}$ and $\left|\nabla\left(\chi^{2} R m\right)\right|_{q}$ are uniformly bounded.

- $\forall q>100,\left|\chi^{2} R m\right|_{\infty}$ and $|\nabla \chi|_{\infty}$ are uniformly bounded.

- The Sobolev constant $A$ is uniformly bounded.

Then $\forall m \geq 0,\left|\nabla^{m}\left(\chi^{2} R m\right)\right|_{\infty}$ is uniformly bounded on $[0, T)$.

Proof. Since Sobolev constant A is assumed to be uniformly bounded on $[0, T)$, then it suffices to prove: $\forall p>100, m \geq 2,\left|\nabla^{m}\left(\chi^{2} R m\right)\right|_{p}$ is uniformly bounded on $[0, T)$. We will prove this by induction.

Assume that : $\left|\nabla^{l}\left(\chi^{2} R m\right)\right|_{p}$ is uniformly bounded on $[0, T), \forall p>100, l \leq m-1$, we should show $\left|\nabla^{m}\left(\chi^{2} R m\right)\right|_{p}$ is uniformly bounded on $[0, T), \forall p>100$.

The induction hypothesis, Lemma 4.6. Proposition 4.13 and bound of Sobolev Constant tells us that:

- $\left|\nabla^{l}\left(\chi^{2} R m\right)\right|_{\infty}$ is uniformly bounded on $[0, T), l \leq m-2$.

- $\left|\nabla^{k} \chi\right|_{p}$ is uniformly bounded on $[0, T), \forall p>100, k \leq m$.

- $\left|\nabla^{j} \chi\right|_{\infty}$ is uniformly bounded on $[0, T), j \leq m-1$.

- $\left|\nabla^{i} R m\right|_{p}$ is uniformly bounded on $[0, T), p>100, i \leq m-1$. 
We compute $\forall p$ :

$$
\begin{aligned}
& \frac{\partial}{\partial t} \int\left|\nabla^{m}\left(\chi^{2} R m\right)\right|^{p} \\
\leq & -\int \chi^{2}\left|\nabla^{m+1}\left(\chi^{2} R m\right)\right|^{2}\left|\nabla^{m}\left(\chi^{2} R m\right)\right|^{p-2} \\
+ & \sum_{i+j+k=m+2, k \leq m+1} \int\left|\nabla^{m}\left(\chi^{2} R m\right)\right|^{p-2}<\nabla^{m}\left(\chi^{2} R m\right), \nabla^{i} \chi * \nabla^{j} \chi * \nabla^{k}\left(\chi^{2} R m\right)> \\
+ & \sum_{i+j+k=m, k \leq m-1} \int\left|\nabla^{m}\left(\chi^{2} R m\right)\right|^{p-2}<\nabla^{m}\left(\chi^{2} R m\right), \nabla^{i}\left(\chi^{2}\right) * \nabla^{j}\left(\chi^{2} R m\right) * \nabla^{k} R m> \\
+ & \int\left|\nabla^{m}\left(\chi^{2} R m\right)\right|^{p-2}<\nabla^{m}\left(\chi^{2} R m\right), \chi^{2} R m * \nabla^{m}\left(\chi^{2} R m\right)> \\
\leq & 1+C \int\left|\nabla^{m}\left(\chi^{2} R m\right)\right|^{p}+\Gamma_{1}+\Gamma_{2}+\Gamma_{3}+\Gamma_{4}
\end{aligned}
$$

In which:

$$
\begin{aligned}
& \Gamma_{1}=\int\left|\nabla^{m}\left(\chi^{2} R m\right)\right|^{p-2}<\nabla^{m}\left(\chi^{2} R m\right), \chi \nabla^{m+2} \chi * \chi^{2} R m> \\
& \Gamma_{2}=\int\left|\nabla^{m}\left(\chi^{2} R m\right)\right|^{p-2}<\nabla^{m}\left(\chi^{2} R m\right), \nabla \chi * \nabla^{m+1} \chi * \chi^{2} R m> \\
& \Gamma_{3}=\int\left|\nabla^{m}\left(\chi^{2} R m\right)\right|^{p-2}<\nabla^{m}\left(\chi^{2} R m\right), \chi * \nabla^{m+1} \chi * \nabla\left(\chi^{2} R m\right)> \\
& \Gamma_{4}=\int\left|\nabla^{m}\left(\chi^{2} R m\right)\right|^{p-2}<\nabla^{m}\left(\chi^{2} R m\right), \chi \nabla \chi * \nabla^{m+1}\left(\chi^{2} R m\right)>
\end{aligned}
$$

Notice $\Gamma_{4} \leq C \int\left|\nabla^{m}\left(\chi^{2} R m\right)\right|^{p}+\frac{1}{16} \int \chi^{2}\left|\nabla^{m+1}\left(\chi^{2} R m\right)\right|^{2}\left|\nabla^{m}\left(\chi^{2} R m\right)\right|^{p-2}$.

When we have applied integration by parts to $\Gamma_{1}, \Gamma_{2}, \Gamma_{3}$ we can control the terms in the way we control $\Gamma_{4}$ except the following term:

$$
\int\left|\nabla^{m}\left(\chi^{2} R m\right)\right|^{p-2}<\nabla^{m}\left(\chi^{2} R m\right), \nabla^{2} \chi * \nabla^{m} \chi * \chi^{2} R m>
$$

But we have assumed $\left|\nabla^{2} \chi\right|_{q}$ is uniformly bounded on $[0, T), \forall q>100$. Then we are done! Actually this is why we should bound $\left|\nabla^{2} \chi\right|_{q}$ first : If $m=2$, then the above term contain $\left|\nabla^{2} \chi\right|^{2}$ then we should deal with the interplay between $\left|\nabla^{2} \chi\right|_{q}$ and $\left|\nabla\left(\chi^{2} R m\right)\right|_{q}$.

Proof. of Theorem 4.1; It is directly implied by Lemma 4.10, 4.11, 4.13, Proposition 4.9, 4.3, and 4.14 .

\section{Proof of the Smooth Extension Part of Theorem 6.3}

We only compute the gap constant in Assumption 2 of Theorem 1.2 and apply Theorem 4.1 , the rest are the same as the computation from page 98 to page 102 of [4].

Notice that: 
- $\left|<R m^{2}+R m \times R m, R m>\right| \leq 5|R m|^{3}$

- $|R| \leq \sqrt{\frac{n(n-1)}{2}}|R m|$

In which $R m^{2}+R m \times R m=R_{i j p q} R_{p q k l}+2 R_{i p k q} R_{j p l q}-2 R_{i p l q} R_{j p k q}$. Then we compute:

$$
\begin{aligned}
& \frac{\partial}{\partial t} \int|R m|^{\frac{n}{2}} \\
\leq & \frac{n}{2}\left\{6.5 \int \chi^{2}|R m|^{\frac{n}{2}+1}-\frac{16}{n^{2}} \int\left|\nabla\left(\chi|R m|^{\frac{n}{4}}\right)\right|^{2}\right\}+C(n)|\nabla \chi|_{\infty}^{2} \int|R m|^{\frac{n}{2}} \\
+ & C(n) \int|\nabla \chi|_{\infty}\left|\nabla\left(\chi|R m|^{\frac{n}{4}}\right)\right||R m|^{\frac{n}{4}} \\
\leq & \frac{n}{2}\left\{6.5 \int\left(\chi|R m|^{\frac{n}{4}}\right)^{2}|R m|-\frac{15.5}{n^{2}} \int\left|\nabla\left(\chi|R m|^{\frac{n}{4}}\right)\right|^{2}\right\}+C(n)|\nabla \chi|_{\infty}^{2} \int|R m|^{\frac{n}{2}} \\
\leq & \left.\frac{n}{2}\left\{6.5\left[|R m|_{\frac{n}{2}} \int\left(\chi|R m|^{\frac{n}{4}}\right)^{\frac{2 n}{n-2}}\right]^{\frac{n-2}{n}}-\frac{15}{A^{2} n^{2}} \int\left(\chi|R m|^{\frac{n}{4}}\right)^{\frac{2 n}{n-2}}\right]^{\frac{n-2}{n}}\right\}+C(n)|\nabla \chi|_{\infty}^{2} \int|R m|^{\frac{n}{2}} \\
- & \frac{1}{4 n} \int\left|\nabla\left(\chi|R m|^{\frac{n}{4}}\right)\right|^{2}
\end{aligned}
$$

From the same analysis from page 98 to page 102 of [4], we see if we assume at $t=0$ that:

$$
\left.|R m|_{\frac{n}{2}}\right|_{0} \leq \frac{2}{n^{2} A_{0}^{2}}
$$

and shrink the constant $C(n, p)$ in Theorem 6.3 to be sufficiently small, we have:

$$
\frac{\partial}{\partial t} \int|R m|^{\frac{n}{2}}+\frac{1}{4 n} \int\left|\nabla\left(\chi|R m|^{\frac{n}{4}}\right)\right|^{2} \leq C(n)|\nabla \chi|_{\infty}^{2} \int|R m|^{\frac{n}{2}}
$$

and

$$
n^{2} A^{2}|R m|_{\frac{n}{2}} \leq \frac{15}{6.5}, \text { in }[0, T)
$$

provided

$$
T \leq C(n, p) \min \left[\frac{1}{\left.|\nabla \chi|_{\infty}^{2}\right|_{0}}, A_{0}^{-\frac{2 n}{2 p-n}} K^{-\frac{2 p}{2 p-n}}\right]
$$

Furthermore, in order to fulfill the step of interpolation in the Moser Iteration from page 98 to page 102 of [4], we should compute:

$$
\begin{aligned}
& \frac{\partial}{\partial t} \int \chi^{2}|R m|^{1+\frac{n}{2}} \\
\leq & \left(1+\frac{n}{2}\right)\left\{6.5|R m|_{\frac{n}{2}}\left(\int\left(\chi^{2}|R m|^{\frac{n}{4}+\frac{1}{2}}\right)^{\frac{2 n}{n-2}}\right)^{\frac{n-2}{n}}-\frac{15}{(n+2)^{2}} \int \mid \nabla\left(\left.\chi^{2}|R m|^{\frac{n}{4}+\frac{1}{2}}\right|^{2}\right\}\right. \\
+ & C(n)|\nabla \chi|_{\infty}^{2} \int|R m|^{\frac{n}{2}}
\end{aligned}
$$


if we assume at $t=0$ that:

$$
\left.|R m|_{\frac{n}{2}}\right|_{0} \leq \frac{1}{2 n^{2} A_{0}^{2}}
$$

and shrink the constant $C(n, p)$ in Theorem 6.3 to be even smaller, we have:

$$
\frac{\partial}{\partial t} \int \chi^{2}|R m|^{\frac{n}{2}+1} \leq C(n)|\nabla \chi|_{\infty}^{2} \int \chi^{2}|R m|^{\frac{n}{2}+1}
$$

and

$$
(n+2)^{2} A^{2}|R m|_{\frac{n}{2}} \leq \frac{15}{6.5}, \text { in }[0, T)
$$

Which give us

$$
\int \chi^{2}|R m|^{\frac{n}{2}+1} \leq C(n) A^{-n} t^{-1}\left(t|\nabla \chi|_{\infty}^{2}+1\right)^{2}
$$

Thus the interpolation argument in moser iteration works. Apply parabolic moser iteration from page 98 to page 102 of [4] we see that $\left|\chi^{2} R m\right|_{\infty}$ is uniformly bounded on $[0, T)$. Then the smooth extension part of Theorem 6.3 is impied by Theorem 4.1 .

\section{Appendix}

Here we state several theorems we used:

Theorem 6.1. (Perelman:)

For all $n$ and $\alpha>0$ there exists $\epsilon$ and $\delta$ such that:

If $[g(t), M]$ is a compact solution to Ricci-Flow: $\frac{\partial g}{\partial t}=-2$ Ric,, $0 \leq t \leq(\epsilon r)^{2}$ and when $t=0$ satisfies:

1. $(1-\delta) C_{E} \operatorname{Vol}(\Omega)^{\frac{n-1}{n}} \leq \cdot \operatorname{Vol}(\partial \Omega), \forall \Omega \Subset B_{0}\left(x_{0}, r\right), C_{E}$ is the isoperimetric constant of the Euclidean space.

2. $R \geq-r^{2}$ on $B_{0}\left(x_{0}, r\right)$

Then $|R m|(x) \leq \frac{\alpha}{t}+(\epsilon r)^{-2}$ and $\operatorname{VolB}\left(x, t^{\frac{1}{2}}\right) \geq C(n) t^{\frac{n}{2}}$ whenever $0<t \leq(\epsilon r)^{2}$ and $d_{t}\left(x, x_{0}\right) \leq$ $\epsilon r$.

Theorem 6.2. Deane Yang: For all $n, p>\frac{n}{2}, \eta, \epsilon$ and $K, \exists \omega_{0}$ and $R$ such that $\forall \tau \leq \omega_{0}, \exists R$ such that: If $[B(x, 1), g]$ be an unit ball in a complete $n$-dim Riemannian Manifold which satisfy:

1. $\operatorname{Vol} B_{0}\left(x_{0}, \tau\right) \geq w(n)(\eta \tau)^{n}$

2. $\mid$ Ric $\left.\left.\right|_{-}\right|_{p[B(x, 1)]} \leq K, p>\frac{n}{2}$

Then we have:

$$
(1-\epsilon) 2^{1-\frac{1}{n}} \alpha(n-1) \alpha^{\frac{1}{n}-1}(n) \eta^{n+1} \operatorname{Vol}(\Omega)^{\frac{n-1}{n}} \leq \operatorname{Vol}(\partial \Omega), \forall \Omega \Subset B(x, R) .
$$


Theorem 6.3. Deane Yang:

For all $n \geq 3, p>\frac{n}{2}, \beta \leq \frac{1}{2 n^{2}}$ and positive constants $A, K$ we have :

Let $[g(t), M]$ be a $n$-dim complete Riemannian manifold and $\chi \in C_{0}^{\infty}(M)$. If $[g(t), M, \chi]$ satisfy:

1. $|f|_{\frac{2 n}{n-2}} \leq A_{0}|\nabla f|_{2}, \forall f \in C_{0}^{\infty}(M)$.

2. $|R m|_{\frac{n}{2}[M]} \leq \frac{\beta}{A_{0}^{2}}, \beta \leq \frac{1}{2 n^{2}}$

3. $|R i c|_{p[M]} \leq K, p>\frac{n}{2}$

Then the local Ricci flow:

$$
\frac{\partial g}{\partial t}=-2\left(\chi^{2} R i c\right)
$$

has a smooth solution up to time:

$$
T=C(n, p) \min \left[\frac{1}{\left.|\nabla \chi|_{\infty}^{2}\right|_{0}}, A_{0}^{-\frac{2 n}{2 p-n}} K^{-\frac{2 p}{2 p-n}}\right]
$$

And when $t \in(0, T]$ we have:

1. $\left|\chi^{2} R m\right|_{\infty} \leq \bar{C}(n) t^{-1}\left[\left.t|\nabla \chi|_{\infty}^{2}\right|_{0}+1\right]^{\frac{4}{n}+2} \beta$

2. $\left|\chi^{2} R i c\right|_{\infty} \leq \bar{C}(n, p) A_{0} \frac{n}{p} t^{-\frac{n}{2 p}}\left[\left.t|\nabla \chi|_{\infty}^{2}\right|_{0}+1\right]^{\frac{n}{p}+\frac{2}{p}} K$

\section{References}

[1] Richard S. Hamilton. Three-Manifolds with Positive Ricci Curvature. J. Diff. Geom. 1982.

[2] Richard S. Hamilton. The Formation of Singularities in the Ricci flow, Surveys in Differential Geometry, vol.2, International Press, Cambridge, MA(1995) 7-136.

[3] Grisha Perelman. The Entropy Formula for the Ricci Flow and its Geometric Applications, preprint.

[4] D. Yang. Convergence of Riemannian Manifolds with Integral Bounds on Curvature I, Ann. Sci. Ecole Norm. Sup. (4) 25 (1992), 77-105.

[5] Ye Li. Local Volume Estimate for Manifolds with $L^{2}$-Bounded Curvature, preprint.

[6] Greene, R. E; Wu, H. Lipschitz convergence of Riemannian manifolds. Pacific J. Math. 131 (1988), no. 1, 119-141.

[7] Gallot, Sylvestre. Isoperimetric Inequalities Based on Integral Norms of Ricci Curvature. Astrisque No. 157-158 (1988), 191-216. 
[8] Croke, Christopher B. Some Isoperimetric Inequalities and Eigenvalue Estimates. Ann. Sci. cole Norm. Sup. (4) 13 (1980), no. 4, 419-435.

[9] Angenent, Sigurd; Knopf, Dan. An Example of Neckpinching for Ricci Flow on $S^{n+1}$. Math. Res. Lett. 11 (2004), no. 4, 493-518.

[10] M.Gromov. Paul Levy's Isoperimetrc Inequality, prepublic. I.H.E.S.1980

[11] M.T. Anderson. Convergence and Rigidity of Manifolds under Ricci Curvature Bounds. Invent. Math. Vol 102. 1990. pp. 429-445.

[12] M.T. Anderson. The $L^{2}$ structure of moduli spaces of Einstein metrics on 4-manifolds, Geom.Fucnt.Anal. Vol.2, No.1 (1992)29-89.

[13] J.Cheeger and G.Tian. Curvature and Injective Radius Estimates for Einstein 4Manifolds. Journal of the American Mathematical Society, Volume 19, Number 2, Page 487-525. 